\title{
REFLEXOS DA PANDEMIA DO NOVO CORONAVÍRUS (COVID-19) ALIADO A TRANSTORNOS PSICOLÓGICOS EM ESTUDANTES UNIVERSITÁRIOS
}

DOI: 10.37702/2175-957X.COBENGE.2021.3548

Gabriela de oliveira Brito - gabriela.brito@aluno.ufop.edu.br

Universidade Federal de Ouro Preto

Rua Antonio José Ramos 228

35400-000 - Ouro Preto - MG

Pedro Corcetti Vitor - pedrocorcetti@gmail.com

Universidade Federal de Ouro Preto

Rua Joaquim Camilo Tavares 480

37018-320 - Varginha - MG

Maria Eduarda Vilela Diniz - dudavileladiniz@gmail.com

Universidade Federal de Ouro Preto

Rua João Fernandes Vieira 274

35400-000 - Ouro Preto - MG

GUSTAVO HENRIQUE COSTA SILVA - gushcs93@gmail.com

Universidade Federal de Ouro Preto

Rua Juscelino Kubitschek 490

34515-170 - Sabará - MG

Carlos Alberto Pereira - pereiraufop@gmail.com

Universidade Federal de Ouro Preto

Rua João XXIII 240

35400-000 - Ouro Preto - MG

Resumo: A pandemia do novo Coronavírus vem afetando diversas áreas da sociedade devido ao modo e à velocidade com a qual ela chegou. A classe dos estudantes brasileiros vem sendo prejudicada com a atual situação mesmo com as medidas que foram tomadas para aliviar a impossibilidade de que haja aulas presenciais em escolas e universidades. A repentina mudança no comportamento, exigido pelos órgãos responsáveis pela saúde, e na rotina dos estudantes 
acarretou no surgimento de problemas relacionados à sua saúde mental. Este documento vem com o objetivo de expor tais problemas que vêm sendo enfrentados. Ansiedade e depressão são as duas doenças que foram apontadas, levando em conta que há um aumento no surgimento de casos desde que a pandemia se iniciou. Foi feito um levantamento com alunos universitários para saber como estes estão lidando com os efeitos psicológicos provenientes da pandemia e para saber como eles consideram o seu atual estado emocional.

Palavras-chave: Depressão. Ansiedade. Pandemia. 


\section{REFLEXOS DA PANDEMIA DO NOVO CORONAVÍRUS (COVID-19) ALIADO A TRANSTORNOS PSICOLÓGICOS EM ESTUDANTES UNIVERSITÁRIOS}

\section{$1 \quad$ INTRODUÇÃO}

A pandemia do coronavírus (covid-19) já atingiu cerca de 38 milhões de pessoas no mundo e aproximadamente 5 milhões de casos já foram apresentados no Brasil. Tudo isso vem mudando a dinâmica de vida das pessoas em todas as regiões, tanto no âmbito econômico e político quanto no social. $O$ isolamento coletivo ocasionado por essa enfermidade foi um dos principais fatores que mudaram e acabaram acarretando alguns problemas psicológicos para a sociedade aumentando os casos de depressão e ansiedade no Brasil, sendo este já considerado segundo o país com o maior número de casos segundo dados da Organização Mundial de Saúde.

A Associação Nacional dos Dirigentes das Instituições Federais de Ensino Superior (ANDIFES) aponta que 15\% dos universitários sofrem de depressão segundo dados de 2018, número quase três vezes maior do que a média nacional, isso em razão da pressão por um bom desempenho e insegurança de um futuro desconhecido. À vista disso foi gerando vulnerabilidade, dificuldade de concentração, cansaço e irritabilidade.

O surto provocou a paralisação das aulas presenciais em todas as universidades de ensino superior do mundo, mudando assim drasticamente a rotina de todos discentes, tanto no modo de relacionar com os professores e colegas de classe como também no modo de aprendizado. Este último citado agora foi de forma remota, uma vez que a forma mais rápida de acesso foram os meios tecnológicos. Com tudo isso, o número de casos de depressão e ansiedade acabou disparando fazendo ligar um alerta para a saúde mental dos estudantes.

Nesse estudo pretende-se analisar os níveis de ansiedade, depressão e estres se em estudantes universitários de forma a comparar a qualidade mental antes e durante a pandemia do novo coronavírus.

\section{METODOLOGIA}

Existem vários transtornos psicológicos que podem ser observados antes e durante algum acontecimento de grande impacto para a sociedade. E em sua grande maioria são considerados como sinais de alerta a presença de algum tipo de conflito interno. A quebra da rotina juntamente com a paralisação das aulas forçou algumas pessoas a ressignificar suas ações no campo universitário, o que trouxe pontos negativos e positivos para elas.

\subsection{Ansiedade}

A ansiedade é um sentimento que pode ser considerado comum para o ser humano. Uma emoção natural proveniente do sistema nervoso em momentos tidos como incomuns no cotidiano. No entanto, quando se trata de uma intensidade elevada, ou seja, níveis alterosos, pode ser vista como uma doença intitulada "Transtorno de Ansiedade Generalizada", que são distúrbios que afetam negativamente a qualidade de vida das pessoas e das demais que estão em seu entorno. Essa emoção pode também acarretar outros males como, por exemplo, a síndrome do pânico, depressão e transtorno obsessivo compulsivo (TOC). 
Baseada em uma visão do senso comum, ansiedade é viver um futuro incerto, causando antecipação de sentimentos desnecessários, tais como medo, preocupação ou temor de situações que podem ou não sair da forma que desejam. A falta de controle de estímulos pode se manifestar tanto no comportamento físico quanto no psicológico. Naturalmente, a ojeriza do porvir e a pressão para um bom desempenho no âmbito educacional deixam os universitários cada vez mais vulneráveis e expostos a doenças com características de fenômenos mentais e essas enfermidades são constantes no meio acadêmico.

A pandemia do (Covid-19), originada pelo vírus SARS-Cov-2 ou comumente chamado de coronavírus, trouxe diversos impactos não apenas na área da biomedicina e epidemiológica como também na área social, econômica, política, cultural e educacional. Não tem como estimar com precisão qual espaço foi mais atingido pelo momento atípico, porém as consequências desse fato trouxeram muitos aspectos negativos para a educação, levando em consideração o desenvolvimento dos discentes na universidade.

A educação foi uma das áreas mais atingidas pela pandemia do novo coronavírus (Covid-19) no Brasil. Com a migração do ensino presencial para o on-line e o estresse causado pelo medo da doença e o possível não aproveitamento do conteúdo pedagógico podem influenciar negativamente na saúde mental dos educandos. (FERMAU,2020.)

\subsection{Depressão}

Conhecida como a doença do século XXI, a depressão é uma doença psiquiátrica caracterizada pela alteração de humor, associada aos sentimentos de dor, desesperança, baixa autoestima, assim como, a distúrbios de apetite e sono, seus sintomas são variados e não se resumem apenas aos citados anteriormente. Suas primeiras observações ocorreram na Idade Média, mas apenas no século XV o termo depressão passou a ser usado e ele designava um estado de desânimo ou perda de interesse. Com o passar do tempo e o aperfeiçoamento da psicologia e psiquiatria, o termo depressão passa a ser amplamente utilizado. Com o desenvolvimento de inúmeras pesquisas, logo, foram descobertos os diversos sintomas que ela provoca e suas causas. Em vista disso, foram observados padrões em tal distúrbio e a partir daí a conclusão de um diagnóstico rápido, comparado ao das décadas passadas, que deve ser realizado apenas por um profissional capacitado na área.

A experiência de ingressar em uma universidade é algo novo para a maior parte dos novos alunos, e com isso, surge um período completo de transformações sejam físicas, psíquicas, afetivas ou sociais. Os discentes vivenciam situações de estresse, cansaço, entre outros. Diante disso, é quase que comum os sintomas da depressão estarem presentes, por algum período, na vida do graduando. Os sintomas afetam diversas áreas da vida de um estudante, como por exemplo o desempenho escolar e nas relações familiares e af etivas. Logo, é indispensável a procura de um médico para um diagnóstico certo, a fim de não se enganar com outras doenças psíquicas.

Com o surgimento do SARS-Cov-2, o mundo entrou rapidamente em uma pandemia, causando o fechamento de escolas, universidades e tudo que gerava alguma aglomeração. Posteriormente, muitos foram perdendo as esperanças do que seria o futuro e foram aos poucos perdendo o interesse em manter sua nova rotina, assim como, entrando em um estado de desânimo constante. $O$ fato de a vida acadêmica estar imprevisível em um momento como essa intriga tanto os docentes como discentes, tudo se torna instável e o medo assombra grande parte dos estudantes. Sentimentos como esse vão absorvendo 
toda saúde mental, e permanecer nisso trará um diagnóstico certo de uma doença psíquica e sérios transtornos causados por ela no futuro.

De acordo com a OPAS - Organização Pan-Americana de Saúde - estudos mostram um aumento significativo em sentimentos como angústia, ansiedade e depressão. Somadas às questões consumo excessivo de álcool, abuso de substâncias e sentimento de perda, tornam-se fatores suficientes para o risco de uma pessoa decidir tirar a própria vida.

\subsection{Pesquisa qualitativa}

Foi realizado um procedimento de pesquisa qualitativa e sistemática que tem como escopo proporcionar respostas às perguntas realizadas em um formulário elaborado no Google Forms e disponibilizadas para o público-alvo entre os dias 12 e 13 de outubro, com o assunto "Ansiedade e Depressão nas Universidades antes e depois da pandemia do novo Coronavírus". A pesquisa desenvolveu-se por um processo constituído de várias perguntas, as quais estão sendo analisadas desde a caracterização do problema tratado até a apresentação em discussão dos resultados. Vejamos todas as perguntas disponibilizadas:

a) Como você considera sua saúde emocional? ( ) Excelente; ( ) Boa; ( ) Regular; ) Regular; ( ) Péssima;

Sim; ( ) Não;

b) Você acha que após seu ingresso na universidade sua saúde mental mudou? ( )

c) Você foi diagnosticado com alguma doença psiquiátrica durante sua graduação? ( ) Sim, depressão; ( ) Sim, ansiedade; ( ) Sim, bipolaridade; ( ) Não; ( ) Outro;

d) Você foi diagnosticado com alguma doença psiquiátrica durante a pandemia? ( ) Sim, depressão; ( ) Sim, ansiedade; ( ) Sim, bipolaridade; ( ) Não; ( ) Outro.

\section{RESULTADOS}

Ao todo, 85 pessoas participaram da investigação e o estudo foi considerado 100\% das respostas dadas pelos entrevistados, como mostra os Gráficos 1, 2 e 3.

Gráfico 1 - Como você considera sua saúde emocional? (Acervo: Google Forms ,2020)

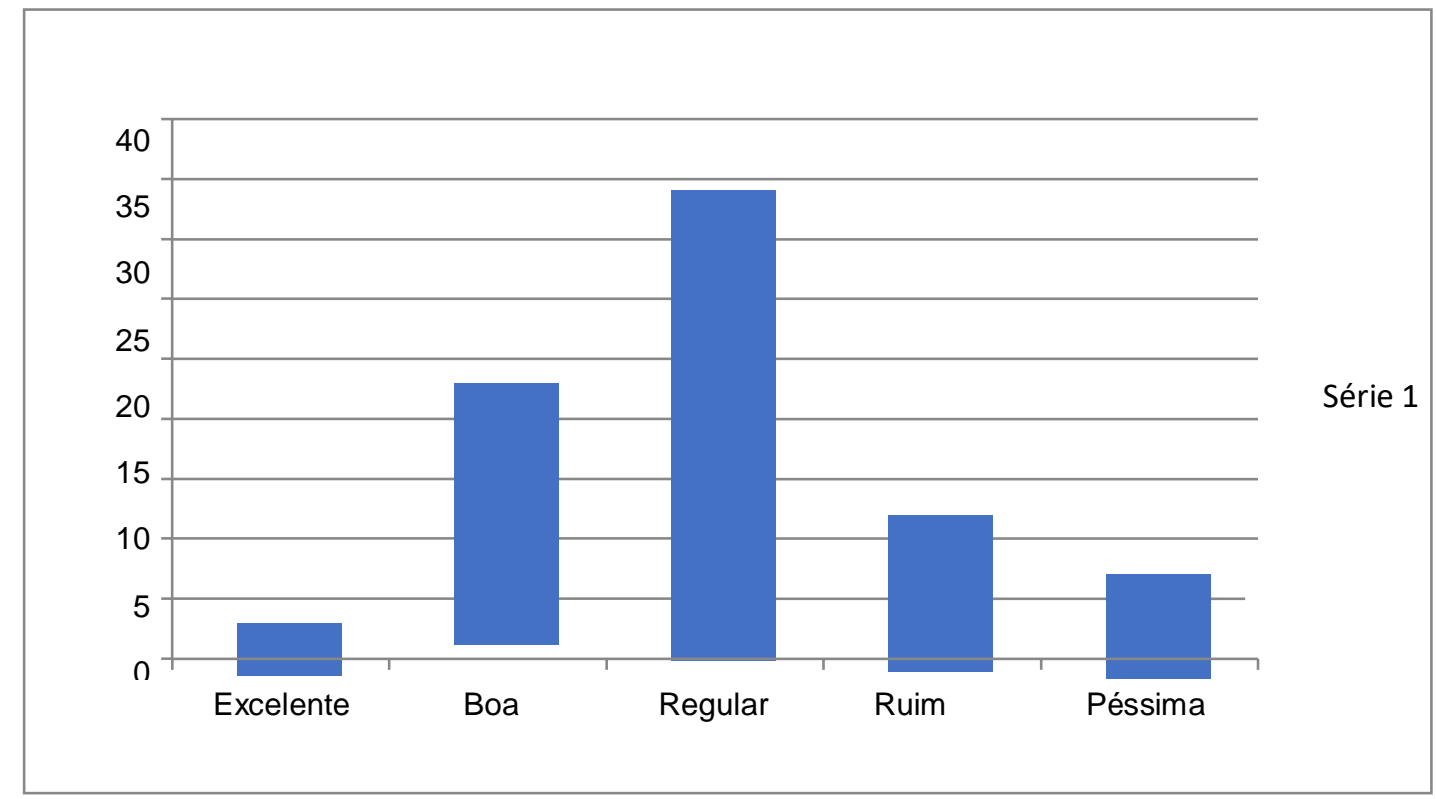

Fonte: Próprio autor 
Gráfico 2- Você acha que após seu ingresso na universidade sua saúde mental mudou? (Acervo:

Google Forms ,2020)

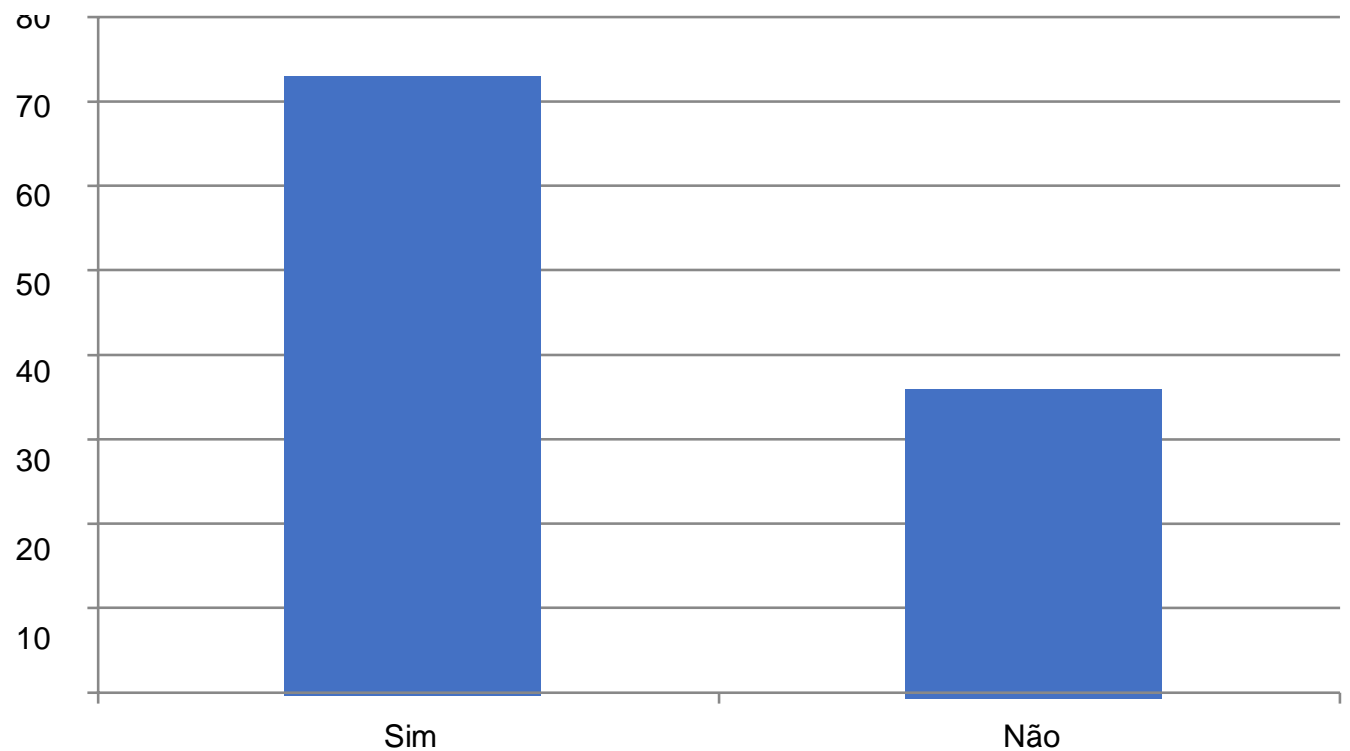

Série 1

Fonte: Próprio autor

Gráfico 3- gráfico comparativo com as perguntas "c" e "d": Você foi diagnosticado com alguma doença psiquiátrica durante sua graduação/ Você foi diagnosticado com alguma doença psiquiátrica durante a pandemia? (Acervo: Google Forms ,2020)

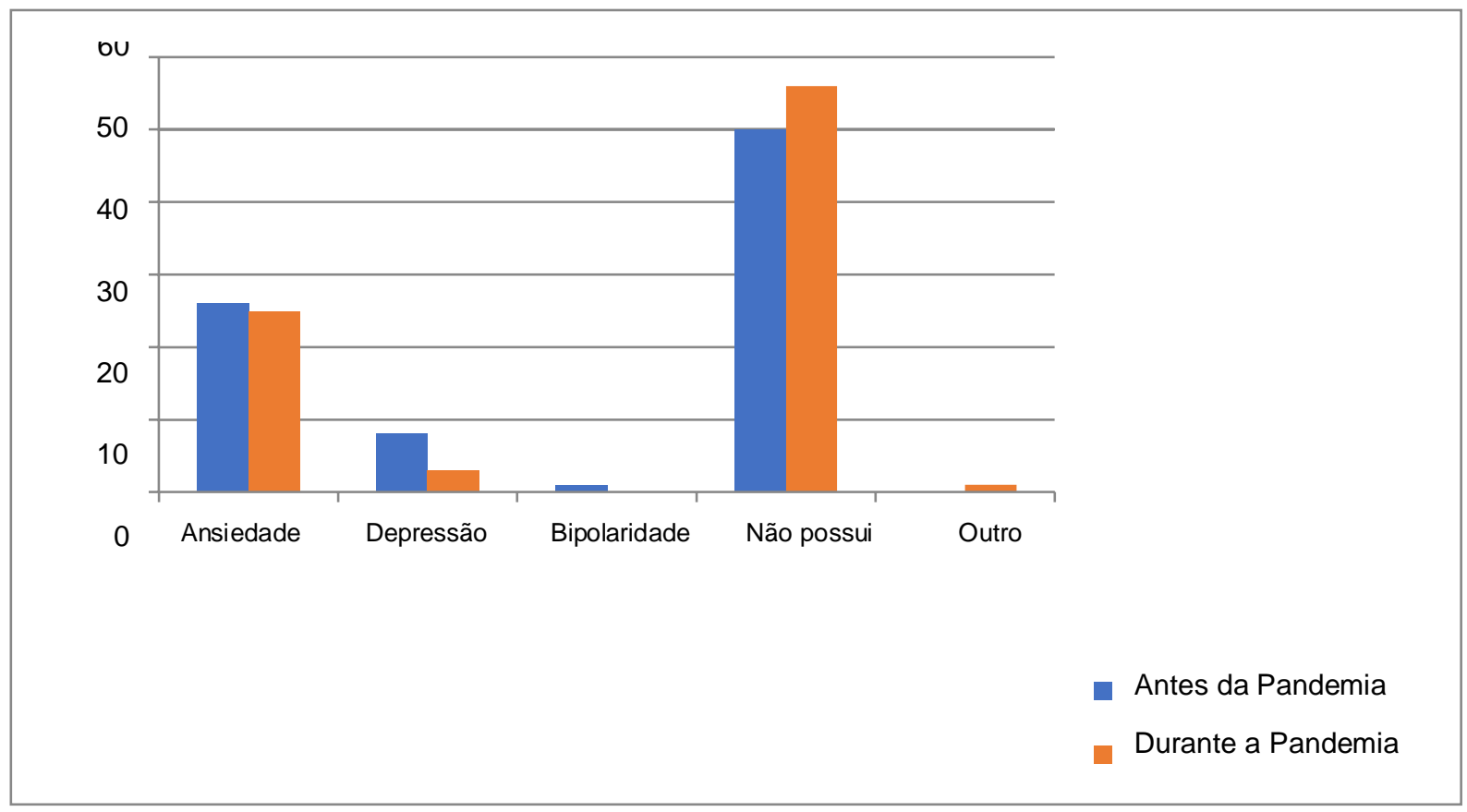

Fonte: Próprio autor 
É fato que a ausência de uma rotina intensifica de maneira séria os sintomas de uma pessoa com ansiedade. Logo, o mal planejamento das aulas e atividades no período remoto se tornou um agravante para os problemas relacionados à ansiedade e depressão enfrentados pelos alunos. Algo tão repentino adicionado em suas rotinas ou a falta delas acabou se tornando um obstáculo a mais a ser afrontado. A dificuldade de comunicação com professores fere ainda mais a situação. Já que, a falta ou o não planejamento das aulas se deve a instituição e os docentes tais alunos não disponham do controle deste cenário, isso pode levar a situação para algo complicado, visto que mesmo com ajuda médica, pessoas que sofrem com essa doença psíquica, principalmente a ansiedade, não atingem o controle da mesma, já que, o meio em que elas estão não contribui para tal.

Conclui-se que mesmo com uma breve pesquisa, muitos alunos não consideraram sua saúde mental saudável. Além disso, mais de $80 \%$ dos alunos relataram sentir sua saúde mental alterar após ingressar na universidade. O Gráfico 3 mostra como o índice de doenças psíquicas entre os estudantes foi alto. Nele foi possível comparar o número de casos antes e durante a pandemia.

\section{CONSIDERAÇÕES FINAIS}

Percebe-se, portanto, que a pandemia vem trazendo muitos desafios que devem ser tratados com zelo. Um grande aumento no número de casos de estudantes com transtornos psicológicos ocorreu nesse período pandêmico. Ansiedade e depressão são assuntos que devem ser expostos a fim de amenizar as suas consequências àqueles que as possuem ou acabaram por adquirir nesse período de adaptações e mudanças na rotina. Esta mudança repentina do modo de viver dos estudantes trouxe consigo a preocupação com seus futuros acadêmicos, dificuldades com o ensino remoto, falta de contato com pessoas próximas, ou seja, muitas barreiras subitamente colocadas para o combate ao Coronavírus, mas que acabam por afetar a sanidade mental de muitos, como foi visto nos resultados da pesquisa realizada com universitários.

Apesar de pouco divulgado, o CVV- Centro de Valorização da Vida - exerce um papel de apoio emocional e prevenção ao suicídio, oferecida pelo Estado. Ela é reconhecida como Utilidade Pública Federal desde 1973. Tal associação civil atende de forma voluntária e gratuita todos que desejam conversar, sob total sigilo por telefone, e-mail ou chat 24 horas todos os dias. Este apoio ajuda milhares de brasileiros todos os anos, logo, cabe às Universidades disseminarem ajuda como está já existente em seu ambiente.

Ademais, acredita-se também na importância da intervenção e acolhimento destes alunos por parte dos discentes e professores da Universidade Federal de Ouro Preto, com foco a amenizar o número de pessoas afetadas por esses tipos de males. Baseado nos números apresentados neste artigo, objetiva-se, também dentro da conclusão, a criação de um centro de acolhimento para alunos, com acompanhamento de um psicólogo (a) e/ou psiquiatra oferecido pela instituição, dentro do Departamento de Engenharia de Minas, no qual será abordado com mais detalhamento em um outro artigo. 


\section{Agradecimentos}

Figueiredo.

Universidade Federal de Ouro Preto, Caio César Gonzaga Alves e Thiago Duarte

\section{REFERÊNCIAS}

CAMARGO,Marcelo. Estudo busca voluntários para avaliar impactos da pandemia na saúde mental. 30 de junho 2020.

Sitedisponivel em<https://ufmg.br/comunicacao/noticias/estudo-busca-voluntariospara-avaliar-impactos-da- pandemia-na-saude-mental>Acesso em 12 out. 2020

\section{FERMAU, Jakeline.Pandemia impacta diretamente na saúde mental} dos estudantes. Fundect. Matogrosso do Sul:2020.Disponível em: $<$ https://www.fundect.ms.gov.br/pandemia-impacta-diretamente-na-saude-mentaldos- estudantes/>. Acesso em: 12 out.2020.

FERREIRA,Camomila Lira et al.Universidade, contexto ansiogênico? Avaliação de traço e estado de ansiedade em estudantes do ciclo básico.Lagoa Nova.2006.Disponível em: <https://www.scielosp.org/article/csc/2009.v14n3/973-981/pt/>. Acesso em: 12 out.2020.

GONÇALVES, Marcelo. Depressão na antiguidade. 10 abr. 2015. Site disponível em: <https://www.psicologiapompeia.com.br/depressao-na-

antiguidade/\#: :text=Depress\%C3\%A30\%20na\%20idade\%20m\%C3\%A9dia\&text=Apena s\% 20em\%201680\%20surge\%20pela,e\%20substitui\%20a\%20palavra\%20melancolia.>. Acesso em 12 out. 2020.

MERCIER,Matheus.Casos de estresse, ansiedade e depressão disparam em meio à pandemia. 13 de maio 2020. Site disponível em<https://www.em.com.br/app/noticia/bemviver/2020/05/13/interna_bem_viver,1146985/casos-de-estresse-ansiedade-edepressao- disparam-em-meio-a-pandemia.shtml>Acesso em 12 out. 2020

O CVV. Disponível em: <https://www.cvv.org.br/o-cvv/>. Acesso em 15 out. 2020.

Pandemia de COVID-19 aumenta fatores de risco para suicídio. 19 set. 2020.

Site disponível em: <https://www.paho.org/pt/noticias/10-9-2020-pandemia-covid-19aumenta- fatores-risco-para-suicidio>. Acesso em 12 out. 2020.

PEIXOTO, Guilherme. Pesquisa da Uerj mostra que casos de ansiedade e estresse aumentaram durante a pandemia do coronavírus.11 agos. 2020. Site disponível em<https://g1.globo.com/rj/rio-de-janeiro/noticia/2020/08/11/pesquisa-dauerj-mostra-que- casos-de-ansiedade-e-estresse-aumentaram-durante-a-pandemiado-coronavirus.ghtml> Acesso em 12 out. 2020

VARELLA, Drauzio. Doenças e sintomas: Depressão. Site disponível em: $<$ https://drauziovarella.uol.com.br/doencas-e-sintomas/depressao/> . Acesso em 12 out. 2020. 
Viviane. 0 incômodo da 'Incerteza e do 'Quase'. Belem.Blog disponível em: $<$ https://www.cvv.org.br/blog/o-incomodo-da-incerteza-e-do-quase/>. Acesso em 12 out. 2020.

\title{
PANDEMIC REFLEXES OF THE NEW CORONAVIRUS (COVID-19) COMBINED WITH PSYCHOLOGICAL DISORDERS IN UNIVERSITY STUDENTS
}

\begin{abstract}
The pandemic of the new Coronavirus has affected several areas of society due to the mode and speed with which it arrived. The class of Brazilian students has been harmed by the current situation even with the measures that have been taken to alleviate the impossibility of face-to-face classes in schools and universities. The sudden change in behavior, required by the organs responsible for health, and in the routine of the students led to the emergence of problems related to their mental health. This document comes with the aim of exposing such problems that have been faced. Anxiety and depression are the two diseases that have been pointed out, considering that there is an increase in the emergence of cases since the pandemic began. A survey was conducted with university students to find out how they are dealing with the psychological effects of the pandemic and to know how they consider their current emotional state.
\end{abstract}

Keywords: Depression. Anxiety. Pandemic. 